Classification

Physics Abstracts

29.15

\title{
Cavité supraconductrice à hélice pour l'accélération d'ions lourds
}

\author{
G. Ramstein, B. Cauvin et J. P. Fouan \\ Service de Physique Nucléaire, Basse Energie, CEN Saclay, 91191 Gif-sur-Yvette Cedex, France
}

(Reçu le 15 octobre, accepté le 19 mai 1988)

\begin{abstract}
Résumé. - Pour permettre d'augmenter la gamme de masse des ions lourds qui seront produits par l'Accélérateur Supraconducteur du CEN Saclay, des cavités à hélice demi-onde ont été étudiées et réalisées en série. Dans cet article, on donnera les caractéristiques principales de ces cavités; en particulier le facteur de surtension et le gain d'énergie par charge, mesuré sous faisceau de ${ }^{12} \mathrm{C}$. Les principaux avantages de ces nouvelles structures sont essentiellement, l'élargissement de la courbe de facteur de temps de transit, permettant de garder une bonne efficacité pour de faibles vitesses, et la simplicité de réalisation mécanique qui minimise le nombre de soudures. On donnera également les performances atteintes pour la série de cavités qui équipe deux des six cryostats du post-accélérateur. Les premiers faisceaux ont été délivrés au début de l'année.
\end{abstract}

\begin{abstract}
Half-wave helix loaded structures have been designed to increase the mass range of heavy ions which will be accelerated by the CEN Saclay superconducting accelerator. In this paper we shall list the main characteristics of these resonators ; especially, the quality factor and the energy gain per charge measured with $a^{12} \mathrm{C}$ beam. The important advantages of these new structures are the broadness of the transit time factor curve which allows us to accelerate low velocity beams with a sufficent efficiency, and the simplicity of mechanical design which requires only two helix welds. We shall also give the performances achieved by the series of resonators which will be mounted into two of the six machine cryostats. The new superconducting accelerator has provided the first beams at the beginning of this year.
\end{abstract}

\section{Introduction.}

De nombreuses études ont été réalisées dans les années 1950 [1, 2] pour l'utilisation de structures haute fréquence à hélice. Il s'agissait alors d'utiliser l'hélice pour ralentir la vitesse de phase d'une onde électromagnétique, et pour produire un champ électrique axial. En couplant ce champ à un faisceau d'électrons, on pouvait utiliser l'énergie cinétique des électrons - en prélevant de l'énergie au faisceau - pour amplifier l'amplitude de l'onde. Ce type de fonctionnement correspond aux tubes à onde progressive [3]. Ce n'est que beaucoup plus tard, dans les années 1960 que fut envisagée la possibilité d'utiliser des structures à hélice pour, à l'inverse, se servir de l'énergie de l'onde HF pour accélérer des faisceaux d'ions. On cherchait alors à augmenter l'énergie des faisceaux d'ions lourds produits par des accélérateurs du type Van de Graaff-Tandem, en construisant des post-accélérateurs utilisant des cavités $\mathrm{HF}[4,5]$. Les premières cavités accélératrices pour ions lourds utilisèrent d'autres dispositifs susceptibles de ralentir la vitesse de phase de l'onde pour l'adapter à la vitesse du faisceau d'ions à accélérer tels que, les cavités réentrantes (Stanford [6] et Darmstadt), ou les cavités à spirale (Heidelberg) [7, 8, 9]. Pour ces cavités fonctionnant à température ambiante, le paramètre essentiel est l'impédance shunt. Il s'agit d'atteindre de forts champs accélérateurs, tout en limitant les pertes par échauffement de la structure. Dès que les premiers laboratoires ont commencé à construire des cavités supraconductrices fonctionnant à la température de l'hélium liquide, les critères de choix d'élaboration des structures furent différents.

En particulier, les cavités à hélice semblaient bien adaptées parce que, d'une part leur structure géométrique permettait un refroidissement aisé, et d'autre part, leur faible encombrement facilitait également leur mise en œuvre. Cependant, on a longtemps pensé que l'hélice n'offrirait pas une stabilité mécanique compatible avec les très fortes valeurs du coefficient de surtension $Q$ des cavités supraconductrices qui imposent à la structure une bande passante très étroite. Des premières études furent développées conjointement par les équipes de Karlsruhe et 
d'Heidelberg [9, 10, 11]. Ces travaux furent poursuivis au CEN Saclay pour aboutir à la construction d'un accélérateur supraconducteur modulaire équipé de 48 cavités à hélice réparties en six cryostats. Chacun d'eux comporte 8 cavités et 2 solénoïdes, également supraconducteurs, permettant de compenser l'effet légèrement défocalisant d'une cavité accélératrice dans le plan radial.

\section{Spécificités des cavités supraconductrices.}

Suivant qu'une cavité accélératrice fonctionne à température ambiante $(300 \mathrm{~K})$ ou à la température de l'hélium liquide (cavités supraconductrices), les critères qui guident les choix, portant sur la géométrie des structures ou la fréquence de résonance, seront très différents.

A température ambiante, de telles structures ne peuvent fonctionner pour de forts champs accélérateurs qu'en régime pulsé. C'est le cas par exemple à Heidelberg [7] où le cycle utile n'est que de $25 \%$ lorsqu'on travaille au champ maximum.

Le paramètre qu'on cherche à optimiser est l'impédance shunt définie par:

$$
R=\left(\frac{\Delta W}{e}\right)^{2} \times \frac{1}{P}
$$

où $\Delta W$ est le gain d'énergie par charge exprimé en $\mathrm{MeV}$;

$P$ est la puissance totale dissipée dans la cavité.

Il s'agira donc d'obtenir le champ accélérateur axial le plus élevé possible pour de faibles pertes.

Pour des cavités supraconductrices, les limitations ne proviennent plus de la puissance dissipée dans la structure mais des champs él $r$.iques et magnétiques maximum admissibles à la surface du matériau. En effet de trop fortes valeurs du champ électrique entraînent, compte tenu de l'état de surface des matériaux, une émission électronique intense qui perturbe notablement le fonctionnement supraconducteur de la cavité. Par ailleurs, des champs magnétiques élevés engendrent des courants de Foucault dans les impuretés conductrices du matériau. Ces courants peuvent créer un point chaud susceptible de réchauffer, par effet Joule, une partie de la structure. Il conviendra donc, cette fois, d'optimiser la géométrie de la structure pour obtenir une distribution du champ électromagnétique qui réduise les rapports $E_{\mathrm{p}} / E_{\mathrm{a}}$ et $B_{\mathrm{p}} / E_{\mathrm{a}}$, c'est-à-dire, qui permette d'obtenir de fortes valeurs du champ accélérateur $E_{\mathrm{a}}$ tout en limitant le champ électrique et magnétique maximum à la surface du matériau $\left(E_{\mathrm{p}}\right.$ et $\left.B_{\mathrm{p}}\right)$ à des valeurs compatibles avec un fonctionnement supraconducteur (Tab. I). Un autre aspect important est la simplicité mécanique de la structure, qui à la fois permet une reproduction aisée, et évite de trop nombreuses opérations de soudage. En effet, lorsqu'une soudure se situe dans
Tableau I. - Propriétés mécaniques et électriques des cavités supraconductrices à hélice.

[Mechanical and electrical properties of superconducting helix loaded cavities.]

\begin{tabular}{|c|c|}
\hline \multicolumn{2}{|l|}{ Caractéristiques mécaniques } \\
\hline $\begin{array}{l}\text { Longueur de la structure (+ tube fais } \\
\text { ceau) }\end{array}$ & $330 \mathrm{~mm}$ \\
\hline $\begin{array}{l}\text { Longueur de l'espace accélérateur équi } \\
\text { valent }(g)\end{array}$ & $80 \mathrm{~mm}$ \\
\hline $\begin{array}{l}\text { Distance entre les centres des espace } \\
\text { accélérateurs }(d)\end{array}$ & es $120 \mathrm{~mm}$ \\
\hline Diamètre de l'enceinte & $225 \mathrm{~mm}$ \\
\hline Diamètre des tubes faisceau & $40 \mathrm{~mm}$ \\
\hline Diamètre du tube de l'hélice & $15 \mathrm{~mm}$ \\
\hline Longueur développée de l'hélice & $303 \mathrm{~mm}$ \\
\hline \multicolumn{2}{|l|}{ Caractéristiques électriques } \\
\hline Fréquence de résonance $(F)$ & $81 \mathrm{MHz}$ \\
\hline Champ accélérateur moyen $\left(E_{\mathrm{a}}\right)$ & $2,15 \mathrm{MV} / \mathrm{m}$ \\
\hline Champ électrique maximum $\left(E_{\mathrm{p}}\right)$ & $16 \mathrm{MV} / \mathrm{m}$ \\
\hline Champ magnétique maximum $\left(B_{\mathrm{p}}\right)$ & $700 \mathrm{G}$ \\
\hline Facteur de surtension $\left(Q_{0}\right)$ & $2 \times 10^{8}$ \\
\hline Facteur géométrique $(G)$ & $9 \Omega$ \\
\hline Gain d'énergie par charge $(\Delta W / q)$ & $535 \mathrm{keV}$ \\
\hline Vitesse d'accélération optimum $\left(V_{\mathrm{opt}}\right)$ & $0,085 \mathrm{C}$ \\
\hline
\end{tabular}

une zone de fort champ magnétique, toute impureté peut devenir la source d'un point chaud, non supraconducteur, qui dissipe la puissance et réchauffe la structure.

\section{Géométrie des cavités demi-onde supraconductri- ces utilisées pour l'accélérateur du CEN Saclay.}

$\mathrm{Au}$ premier ordre, on peut considérer que la distribution de potentiel le long de l'hélice a approximativement la forme d'une arche de sinusoïde (demionde). Les deux extrémités de l'enroulement sont au potentiel zéro ainsi que l'enceinte. Le centre de l'enroulement hélicoïdal est porté au potentiel maximum. Cette distribution conduit à une géométrie complexe de l'hélice pour les raisons suivantes:

- Le centre de l'enroulement étant porté au potentiel maximum et l'enceinte étant au potentiel zéro, on a réduit le diamètre des spires centrales, pour éviter d'atteindre un champ électrique radial trop important (Fig. 1a).

- A chaque extrémité de l'enroulement se développe un fort gradient de potentiel, et donc un fort champ électrique entre spires. Pour limiter ce champ l'écartement entre les spires situées aux extrémités de l'enroulement a été augmenté (Fig. 1a).

L'absence de symétrie d'une telle géométrie ne permet pas d'utiliser les programmes de simulation 


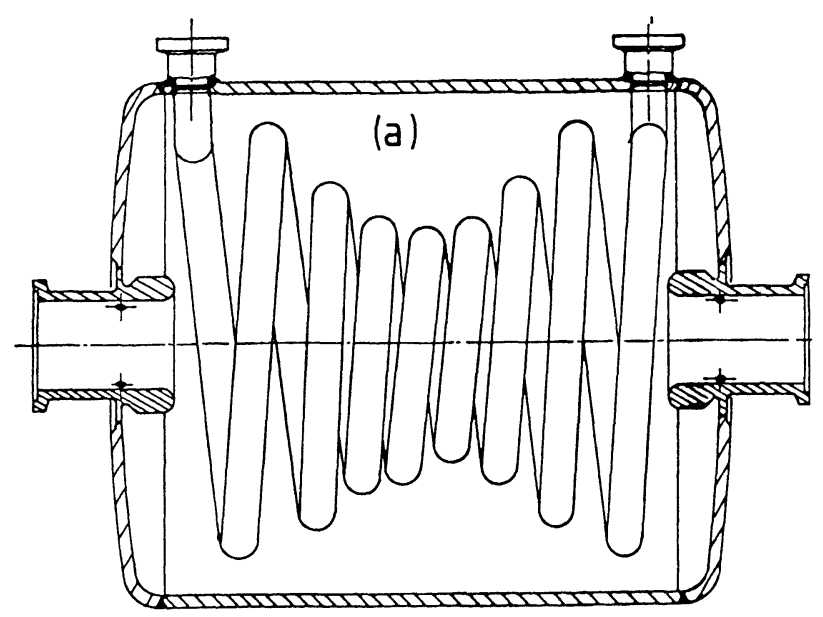

Fig. 1a. - Coupe longitudinale d'une cavité demi-onde.

[Longitudinal section of a half wave resonator.]

classiques pour évaluer avec une précision suffisante la distribution du champ électromagnétique à l'intérieur de la cavité. On a donc utilisé la méthode classique des perturbations $[12,13]$ pour déterminer la localisation, et pour mesurer les valeurs maxima du champ électrique et magnétique. On a également mesuré la distribution du champ électrique accélérateur sur l'axe de la cavité [14] (Fig. 1b).

Comme on peut le constater sur cette figure, le champ accélérateur varie continûment sur l'axe de la cavité. On ne dispose pas, comme dans d'autres structures, d'espaces accélarateurs séparés par des tubes de glissement $[15,16]$, mais plutôt de deux « zones accélératrices ».

La fréquence de résonance est de $81 \mathrm{MHz}$. Elle varie approximativement comme l'inverse de la

E

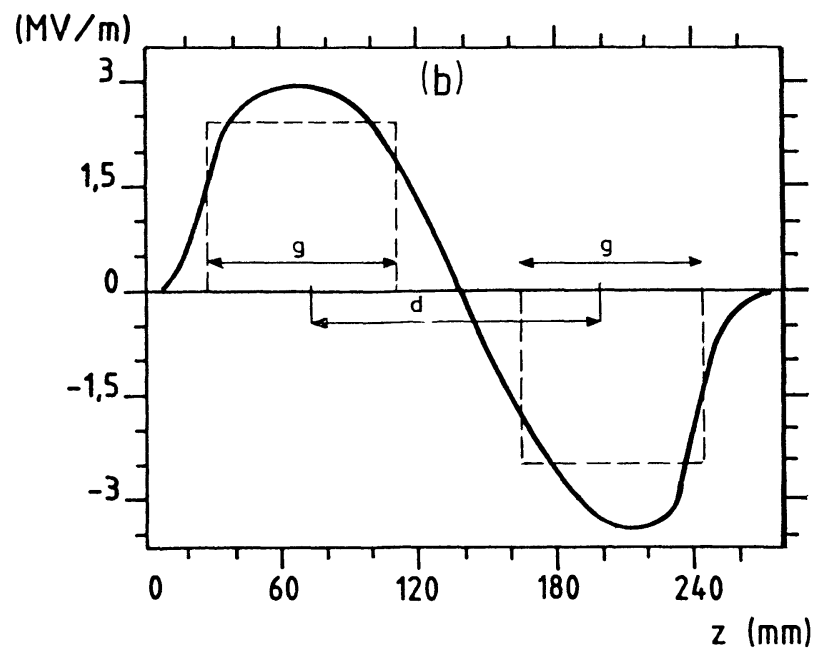

Fig. 1b. - Champ électrique accélérateur sur l'axe de la cavité.

[Electric accelerating field on the resonator beam axis.] longueur développée de l'hélice [17], ce qui nous a permis de modifier ce paramètre jusqu'à obtenir un ensemble (hélice soudée dans l'enceinte) résonnant à $81 \mathrm{MHz} \pm 100 \mathrm{KHz}$. L'avantage principal des cavités à deux espaces accélérateurs par rapport aux cavités à trois espaces accélérateurs ou plus [18, 19] est d'élargir l'acceptance en vitesse de la structure.

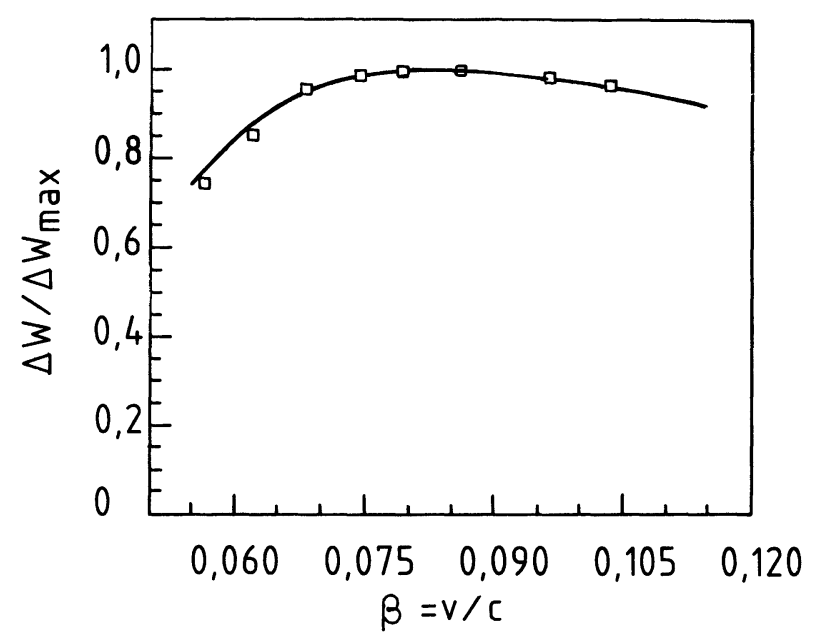

Fig. 2. - Gain d'énergie par charge en fonction de la vitesse du faisceau. Le gain d'énergie est normalisé à 1 . $\left(\Delta W_{\max }=535 \mathrm{keV}\right.$ pour $\left.\beta=0,085\right)$. La vitesse du faisceau est rapportée à la vitesse de la lumière paramètre $\beta=v / c$.

[Energy gain per charge versus beam velocity. The energy gain is normalized, taking into account that $\Delta W_{\max }=$ $535 \mathrm{keV}$ for $\beta=0.085 \mathrm{C}$. The beam velocity is divided by speed of light : parameter $\beta=v / c$.]

On a représenté sur la figure 2, le gain d'énergie par charge normalisé, qu'on peut calculer à partir de la distribution du champ $E(z)$ sur l'axe :

$$
\Delta W=e \int_{0}^{L} E(z) \sin \left(\frac{\omega z}{v(z)}+\phi\right) d z
$$

où $\omega=2 \pi f$ est la pulsation de la cavité ;

$L$ la longueur de la structure;

$E(z)$ le champ électrique sur l'axe;

$v(z)$ vitesse de la particule à l'abscisse $z$;

$\Delta W_{\max }=535 \mathrm{keV}$ par charge.

On peut constater que la cavité a une très bonne efficacité (supérieure à $75 \%$ ) sur une très large plage de vitesse $0,055 \mathrm{C}<\beta<0,12 \mathrm{C}$. En particulier pour les faisceaux les plus lourds délivrés par le Tandem avec une vitesse de $0,05 \mathrm{C}$, la cavité demionde reste encore très efficace.

On a également mesuré le gain d'énergie par charge par diffusion élastique sur une cible d'Or d'un faisceau continu de carbone-12. Les points de mesure reportés sur la figure 2 sont en très bon 
accord avec les calculs théoriques, effectués à partir de la distribution de champ obtenue par la méthode des perturbations.

\section{Test de cavités demi-onde dans l'hélium liquide.}

Le matériau choisi pour fabriquer ces cavités supraconductrices est le niobium massif dont la température critique est de 9,2 K. Ces cavités sont placées dans des cryostats et baignent dans l'hélium liquide bouillant à la pression atmosphérique pour une température de 4,2 K. L'hélice elle-même est refroidie par flux forcé d'hélium.

Le paramètre essentiel qui gouverne les dissipations d'énergie à basse température et donc détermine la puissance du réfrigérateur est le facteur de surtension défini par :

$$
Q=\frac{2 \pi f \cdot W}{P}
$$

où $W$ est l'énergie emmagasinée dans la structure ; et $P$ la puissance totale dissipée.

Ce facteur est de l'ordre de quelques $10^{3}$ pour des structures fonctionnant à température ambiante et de $10^{8}$ à $10^{9}$ pour des cavités supraconductrices. La résistance de surface de ces dernières, pour des fréquences de l'ordre de $100 \mathrm{MHz}$ est réduite à la résistance résiduelle, c'est-à-dire plus faible de 5 ordres de grandeur qu'à température ambiante.

La mesure du facteur de surtension est essentielle, car elle nous donne des informations, d'une part sur la qualité du matériau : inclusion ou impureté à la surface du niobium (la profondeur de pénétration du champ magnétique dans le niobium à $81 \mathrm{MHz}$ est de quelques centaines d'angström), et d'autre part sur l'état de surface : poussière, oxydation, micropointe.

Sur la figure 3 , on a représenté l'évolution du facteur de surtension avec le champ accélérateur moyen. Lors d'un premier test, on note une très nette dégradation du facteur de surtension à partir d'un champ de 1,6 MV/m due à une forte émission électronique (émission froide). Après un polissage chimique et un lavage hors-poussière de la cavité, un second test à froid de la structure a été effectué, pour lequel on constate une nette amélioration de la valeur du coefficient de surtension, et la disparition de l'émission électronique. Le champ maximum atteint est plus élevé : $2,15 \mathrm{MV} / \mathrm{m}$.

Pour pouvoir améliorer les performances d'une cavité, mais également pour tenter de comprendre quel type de processus va conduire à la perte du caractère supraconducteur, on a besoin de connaitre avec précision la source du réchauffement brutal de la cavité. Une telle détermination est possible si on abaisse la température de l'hélium à $1,8 \mathrm{~K}$ ce qui permet d'utiliser la propagation de l'onde de «second son» générée par le réchauffement dans l'hélium superfluide $[14,20]$. On a pu ainsi localiser

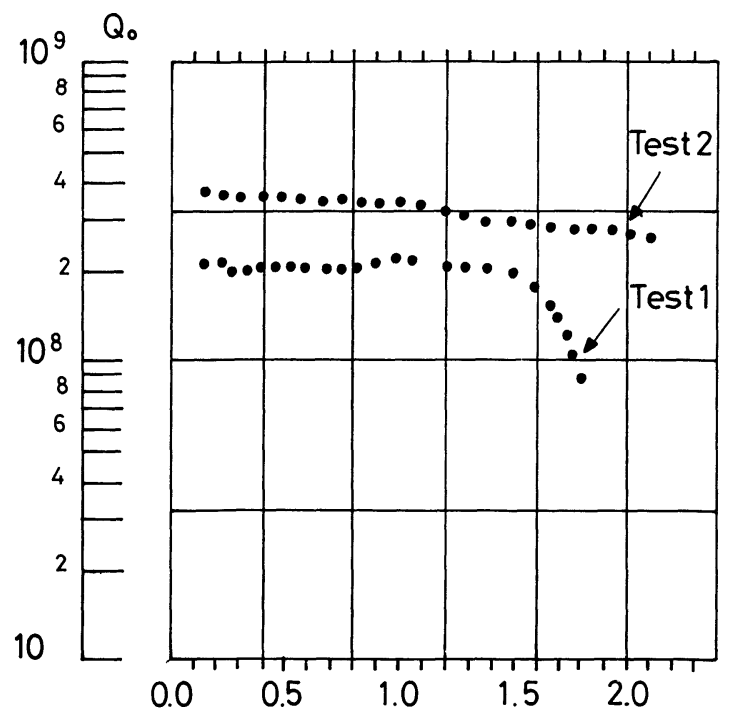

Ea champ accélérateur moyen $(\mathrm{MV} / \mathrm{m})$

Fig. 3. - Evolution du facteur de surtension $Q_{0}$ avec le champ accélérateur moyen $E_{\mathrm{a}}$. Premier test : forte émission électronique à partir de $E=1,6 \mathrm{MV} / \mathrm{m}$ qui conduit à une dégradation du facteur de surtension. Second test après polissage et montage hors poussières : nette amélioration de l'état de surface qui se traduit par une augmentation du facteur de surtension, une disparition de l'émission froide, et un champ accélérateur plus élevé : 2,15 MV/m.

[Quality factor versus electric accelerating field. First test : important field emission for $E_{\mathrm{a}}=1.6 \mathrm{MV} / \mathrm{m}$ which leads to quality factor degradation. Second test, following chemical polishing, cleaning and dustfree mounting. This improves surface quality and leads to higher quality factors ; it cancels field emission, and increases the accelerating field $(2,15 \mathrm{MV} / \mathrm{m})$.]

avec une précision de $\pm 0,5 \mathrm{~cm}$ la position de la source émettrice de l'onde de chaleur. Des mesures systématiques nous ont permis de constater que dans la plupart des cas, ces sources se situent au niveau des soudures de l'hélice dans l'enceinte qui sont également dez zones de forts champs magnétiques et de courants élevés. Il est probable que près des soudures la pureté du matériau soit altérée par le procédé de soudure utilisé (TIG). Des courants de Foucault peuvent alors se développer dans ces zones non supraconductrices qui deviennent des points chauds, dissipent très vite beaucoup d'énergie et réchauffent une partie de la structure en provoquant la perte de la supraconductivité.

Une autre amélioration sensible a pu être réalisée en blindant les cavités contre le champ magnétique terrestre. En effet, on sait que pour un supraconducteur parfait, à la température de transition, le flux du champ magnétique est expulsé du métal : c'est l'effet Meissner. Or, il se trouve que pour un matériau réel, qui contient toujours des impuretés, une partie du flux magnétique est piégée dans les zones qui restent non supraconductrices, donc augmentent les pertes 
et dégradent ainsi le facteur de surtension. Un tel effet a dèjà été mis en évidence à Karlsruhe par B. Piosczyk et al. [21]. Compte tenu du coût de la réfrigération, il importe de diminuer au maximum la puissance dissipée à $4,2 \mathrm{~K}$. La figure 4 montre l'évolution du facteur de surtension en fonction du champ électrique accélérateur. Dans le premier cas, on a refroidi la structure sans utiliser aucune protection contre le champ magnétique ; un champ magnétique de $250 \mathrm{mG}$ a été alors mesuré dans le cryostat. Dans le second cas, on a blindé la cavité avec des plaques de matériau à perméabilité magnétique élevée (type "CO-NETIC») et le champ magnétique résiduel n'est plus alors que de $50 \mathrm{mG}$ lorsqu'on refroidit la cavité.

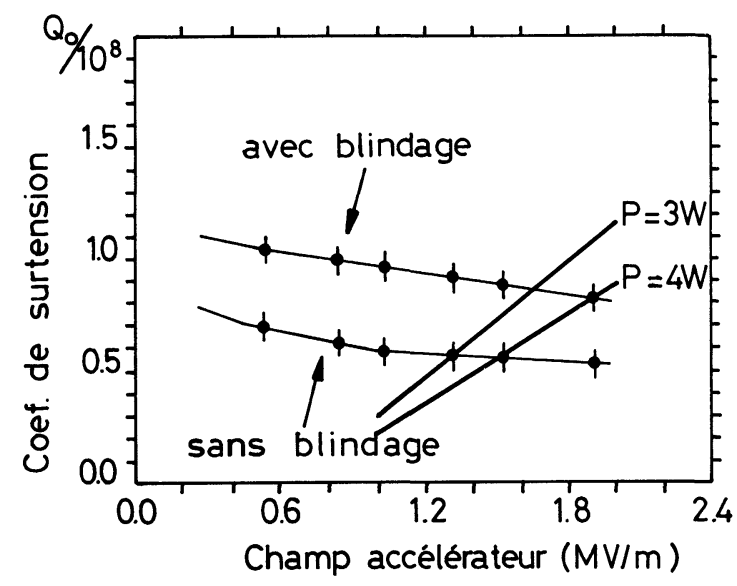

Fig. 4. - Evolution du facteur de surtension avec le champ électrique accélérateur avec et sans blindage contre le champ magnétique terrestre. Les courbes en trait plein représentent les paraboles $Q=\alpha E^{2}$ à puissance constante.

[Quality factor versus electric accelerating field with and without shielding against the earth magnetic field. Curves in solid line show the parabola $Q=\alpha E^{2}$ at constant power.]

On peut constater qu'un tel blindage permet d'augmenter le facteur de surtension, et donc de réduire sensiblement la puissance dissipée à $4,2 \mathrm{~K}$.

Les principales caractéristiques géométriques et électriques de la cavité demi-onde sont résumées dans le tableau I.

\section{Fabrication d'une série de vingt cavités demi-onde pour l'accélérateur supraconducteur.}

Une série de 20 cavités a été réalisée pour équiper deux des six cryostats de l'accélérateur. Dans cette perspective, on a conçu un mandrin d'enroulement pour l'hélice qui a permis de fabriquer des cavités dont la géométrie, et par conséquent la fréquence de résonance, sont parfaitement reproductibles.
D'autre part, à toutes les étapes de la fabrication, nous avons tenu compte de tous les progrès récents effectués en technologie supraconductrice HF. Ceux-ci sont résumés ci-dessous :

L'état de surface est, comme on l'a vu, un paramètre essentiel dans l'élaboration des cavités supraconductrices. Les cavités complètement soudées subissent un traitement chimique, qui est un polissage au trempé, dans un mélange d'acide [22]. Puis elles sont rincées, en utilisant de l'eau hyperpure de forte résistivité $16 \mathrm{M} \Omega . \mathrm{m}$, filtrée (diamètre du filtre $0,2 \mu$ ) dans une salle hors-poussière pour éviter tout dépôt à la surface de l'hélice et de l'enceinte. Elles sont ensuite séchées sous flux laminaire d'air (classe 100 : moins de 100 particules de diamètre supérieur à $0,2 \mu$ par pied cube). On cherche en particulier à éviter que les micro-poussières ne restent dans l'enceinte, en effet celles-ci sont des sources d'émission électronique intense qui dégrade les performances de la cavité. C'est aussi la raison pour laquelle tous les montages se font systématiquement sous flux laminaire d'air. Ce mode opératoire a permis d'obtenir pour l'ensemble des cavités de très bons résultats du point de vue du champ accélérateur moyen (Fig. 5) et du coefficient de surtension.

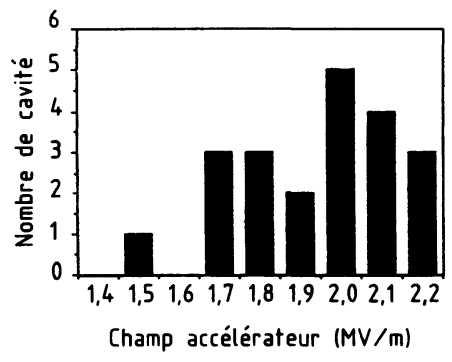

Fig. 5. - Histogramme des champs accélérateurs moyens pour la série de cavités demi-onde.

[Histogram of accelerating fields performances for the series of half-wave resonators.]

\section{Conclusions.}

Ces cavités seront placées en début d'accélération où elles ont leur efficacité maximum. En particulier, leur faible fréquence de travail et leur fort gain d'énergie même à faible vitesse améliorent sensiblement la dynamique des faisceaux les plus lourds, et permettent d'élargir la gamme de masse des ions accélérés. L'accélérateur supraconducteur du CEN Saclay comprend 48 cavités accélératrices, dont 16 cavités demi-onde, et utilise comme injecteur le Tandem de 9 MV. Des faisceaux d'ions lourds (du carbone au germanium) seront délivrés dès le début de l'année 1988. Grâce à sa structure modulaire et à ses très bonnes qualités de faisceaux, des expériences de physique nucléaire fines pourront y être réalisées. 


\section{Bibliographie}

[1] Laplume, J., Onde Electrique 263 (1949) 66.

[2] Lapostolle, P. M., Ann. Télécomm. 12 (1957) 34.

[3] PIerce, J. R., Travelling Wave Tube (D. Van No and Compagny eds, 1950).

[4] Klein, H., et al., Proton Linear Accelerator Conference, Los Alamos (1972).

[5] Sierk, A. J., et al., Part. Accel. 2 (1971) 149.

[6] Ilan Ben Zvi et al., Part. Acc. 7 (1976) 125.

[7] HUCK B., et al., IEEE Trans. Nucl. NS 28 (1981).

[8] ShEPARD, K. W., et al., IEEE Trans. Nucl. NS 22 (3) (1975).

[9] Bollinger, L. M., et al., AECL Report 5677 (95) (1976).

[10] HochsCHILD, G., et al., Rapport KFK 2624 (1978).

[11] Hochschild, G., et al., IEEE Trans. Nucl. NS 24 (3) (1977).

[12] Argence, E. et Kahan, Th., Théorie des guides d'onde et cavités électromagnétiques (Paris, Dunod, 1964).

[13] Slater, J., Microwaves Electronics (Van Nostrand Company Eds, 1963).
[14] Ramstein, G., Note CEA-N-2539 (1987).

[15] Ilan Ben Zvi, Part. Accel. 9 (1979) 169.

[16] Bollinger, M., Ann. Rèv. Nucl. Part. Sci. 36 (1986) 475.

[17] SCHEMPP, A., et al., Nucl. Instr. Meth. (1976) 29.

[18] ZIEHER, K. W., et al., Investigation of a modified helices loaded superconducting resonator for acceleration of heavy ions, KFK Karlsruhe (1980).

[19] Cauvin, B., et al., Proceeding of the third Workshop on RF superconductivity, Argonne, 14-18 septembre 1987.

[20] Huebener, R. P., Magnetic flux structures in supraconductor (Ed. Springer Verlag, Berlin, 1976).

[21] PIOsCZYK, B., et al., IEEE Trans. Nucl. NS 20 (1973) 108.

[22] Bloess, D., Proceedings of the 2nd Workshop on RF supraconductivity, Genève, CERN (1984) 409. 